\title{
Personalizing medicine through targeted agents, capitalizing on pathway dysregulation and
} \section{biomarker use}

American Association for Cancer Research Centennial Conference on Translational Cancer Medicine 2008: Cancer Clinical

Trials and Personalized Medicine, Monterey, CA, USA, 20-23 July, 2008

Kim Marie Hirshfield

UMDNJ/Robert Wood Johnson

The American Association for Cancer Research Centennial Conference on Translational Medicine 2008: Cancer Clinical Trials and Personalized Medicine, held on July 20-23, 2008 , merged research approaches with a unique mix of presenters and attendees from academia, government and industry. The goal was to foster collaborations and discussion between these groups, to advance knowledge in therapeutic effectiveness and direction of biomarker incorporation to personalize therapies and design new therapeutic approaches.

Charles Sawyers, the keynote speaker, addressed the use of targeted agents in chronic myelogenous leukemia and molecular mechanisms of resistance. Relapses with the first-line tyrosine kinase inhibitor, imatinib, occur by mutations in Ablkinase. With more than 50 distinct mutations in imatinib-resistant disease and novel mutations with the second-line agents, ongoing studies of these agents will compare responses to imatinib and durability of response. Compound mutations eventually lead to resistance to both agents and other candidates are being tested for effectiveness. Transient versus continuous inhibition needs evaluation because the former may give maximal anti-tumor benefit.

Limited effectiveness of antiandrogens for prostate cancer is due to resistance through androgen receptor (AR) gene amplification, mutation, or upregulation. The novel small molecule RD162 has higher AR affinity, inhibits growth with no agonistic properties, impairs binding to androgen response elements (AREs), and is active in bicalutamide-resistant prostate cancer models. Prostate-specific antigen (PSA) response data were presented at the American Society of Clinical Oncology (ASCO, Chicago, IL, USA, 30 May-3 June 2008) for a Phase I/ II trial in castrate-resistant prostate cancer, but dose-escalation is ongoing. Kenneth Pienta described how CCL2, elaborated by bone marrow endothelial cells, is upregulated in bone metastases and acts as a monocyte chemoattractant. Presence of tumor-associated monocytes corresponds with prostate cancer progression. While anti-CCL2 antibodies reduce bone metastasis, cell proliferation and osteoclast function in mouse models, the goal will be to combine it with cytotoxins. William Nelson also discussed the role of epigenetic silencing in prostate tumorigenesis. Methyl-CpG binding domain protein 2 (MBD2) interacts with methylated heterochromatin and is needed for tumorigenesis. A small molecule inhibitor interferes with MBD2:methylated DNA binding. As MBD2 inhibition in prostate cancer reactivates retinoid signaling in xenograft models, a trial combining isotretinoin and the MBD2 inhibitor is underway.

Combination therapies delaying or preventing resistance and increasing anti-tumor efficacy require knowledge of cytotoxic activity, toxicities at maximally-tolerated dose (MTD), resistance mechanisms and interactive effects. Chris Takimoto described the use of classical yeast genetics for cancer drug development. Yeast libraries are useful tools for target validation, genome-wide scans for loss-of-function and sensitivity screens to identify novel combinatorial regimens. Although cytotoxic combinations have proven effective in many tumor types, Miguel Villalona-Calero introduced modulation of DNA-damaging agents, such as mitomycin $\mathrm{C}$ in combination with celecoxib or CPT11, showing activity in several solid cancers and heavily pretreated patients. Phase I trials of Poly(ADP-ribose) polymerase (PARP) inhibitors in BRCA1/2 carriers with ovarian cancer take advantage of the principal that DNA repair deficiency confers higher sensitivity to DNA-damaging agents.

Since blockade of one signaling pathway may lead to activation of compensatory pathways, Jose Baselga outlined strategies using nonoverlapping actions and hitting vertical/transverse
Medical School, The Cancer

Institute of New Jersey,

New Brunswick, NJ, USA

Tel.: +1 7322356028

Fax: +1 7322355331

hirshfie@umdnj.edu 
targets including combined receptor and downstream inhibitors. Numerous antibody agents and small-molecule inhibitors for HER1 (panitumamab, EMD-7-2000, h-R3) and HER2 (pertuzumab) are under development. Novel therapies include trastuzumab-DMI, an antibody-drug conjugate, and Hsp90 inhibitors. To overcome trastuzumab-resistant truncated HER2, NeoALTTO is evaluating biomarkers and neoadjuvant trastuzumab and paclitaxel, lapatinib and paclitaxel, or paclitaxel plus lapatinib plus paclitaxel in breast cancer.

Although unexpected, dramatic responses to erlotinib have been observed in nonsmokers with nonsmall cell lung cancer (NSCLC), no survival benefit is seen in combination with cytotoxics. KRAS mutations associated with primary resistance to EGFR tyrosine kinase inhibitors in NSCLC are negative predictors for response to anti-EGFR antibodies in colon cancer $[1,2]$. Charles Sawyers addressed the use of EGFR and mTOR inhibitors in gliomas in a trial through the Cancer Genome Atlas (TCGA) Research Network. EGFR inhibitor-resistant gliomas were treated with mTOR inhibitor prior to and after salvage resection. Only loss of phosphatase and tensin homolog (PTEN) was predictive of response. To prevent feedback activation of IGF-1 with mTOR inhibition, MK-8669 (an mTOR inhibitor) and the anti-IGF1 antibody MK-0646 are being combined with supra-additive and antiproliferative effects in other tumors. Data from Peter Houghton with $p 53$-mutant sarcoma cell lines show CP751,871 (anti-IGF-1 receptor antibody) and rapamycin induce apoptosis and inhibit VEGF secretion.

Branimir Sikic covered cancer drug resistance that is attributable in part to efflux pumps, metabolism, drug uptake and sequestration. While protective in normal tissues, constituitive expression of p-glycoprotein in many cancers, confers drug resistance and is adversely prognostic. Small-molecule inhibitors of p-glycoprotein are the most promising: quinine, ciclosporin and verapamil (nonspecific agents); zosuquidar, tariquidar, biricodar and valspodar (targeted agents). Their use may require dose modifications of combined drugs and monitoring for dose-limiting side effects. A Phase III trial of zosuquidar in secondary acute myelogenous leukemia is underway.

The evolving 'influence of tumor microenvironment on drug response and resistance' was presented by William Dalton with a focus on adhesion in multiple myeloma. $\beta 1$ integrin- and fibronectin-mediated adhesion confers melphalan resistance in myeloma cells. Adhesion enhances stat 3 signaling. The JAK/stat pathway is constitutively activated in myeloma. A peptide was identified that inhibits integrin-mediated adhesion in a dose-dependent fashion with reduced melphalan resistance. In myeloma mouse models, peptide treatment reduces tumor burden both alone and with melphalan. Similarly, $\gamma$ secretase inhibitors, which block the JAK/stat pathway, induce apoptosis and reduce myeloma burden in combination with doxorubicin in mouse models.

Douglas Hanahan reviewed several mechanisms by which resistance develops to antiangiogenic therapy. Adaptive resistance occurs through upregulation of alternative pro-angiogenic signaling that effect non-VEGF-dependent revascularization and tumor regrowth. Blockade of FGF reduces revascularization in human gliobastoma multiforme treated with pan-VEGF blockade by AZD2171. Other mechanisms of resistance are recruitment of proangiogenic myeloid cells, pericyte coverage and increased invasiveness/metastasis. One implication is that potent angiogenesis inhibitors in combination with agents blocking invasion and metastasis may produce more enduring effects.

Joyce Slingerland identified the importance of cellular sarcoma gene (SRC) targeting in breast cancer since aromatase inhibitors increase SRC levels. Anti-estrogens induce p27-mediated cell cycle arrest. Loss of p27 through SRC activation abrogates that arrest and is associated with worse survival. The investigational drug AZD0530 decreased SRC. Combined use of AZD0530 and anastrazole prevented SRC expression and p27 expression increased. Validation in mouse models led to Phase I and neoadjuvant trials for locally advanced breast cancer where pre- and post-treatment biopsies will be performed for marker analysis.

\section{Biomarkers as indicators of response to therapy}

The challenges are great for incorporating biomarkers in studies and are reviewed elsewhere [3]. Ideally, knowing effect on relevant targets and dysregulated pathways would predict drug efficacy. Limitations in performing biomarker studies include lack of tissue, surrogate marker validity, using surrogate tissues, animal models and quality control. Functional molecular imaging studies as surrogate end points may overcome biopsy issues and correlate with clini$\mathrm{cal} /$ anatomic response. Mark Ratain stressed that biomarker development "requires changing our ideas of what markers are", for example, the 
tumor-size-survival relationship. Biomarkers are useful to define inclusion/exclusion criteria for therapeutic interventions and patient selection may be pivotal for identifying targeted agents and improving outcomes and cost-effectiveness. The caveat, however, is that if the wrong population or marker is selected, an otherwise good drug candidate may be discarded. Therefore, biomarker development and use requires good standards, data sharing, genomic mapping and organizational support.

Exemplifying some of these issues is the use of trastuzumab and HER2. Tumor classification is important for selection of study patients and for predicting response to treatment. Diagnostic challenges were significant with trastuzumab but the landscape is evolving in what test to use, for example, immunohistochemistry, fluorescence in situ hybridization (FISH) for HER2 testing, or newer tests, such as heterodimerization assays, HERmark $^{\mathrm{TM}}$. Classifiers may be unexpected as demonstrated by low HER3 being predictive of response to pertuzumab in ovarian cancer.

Many drugs in development fail to enter Phase II trials and get approved. Nicholas Dracapoli presented data supporting the positive impact of companion diagnostics on drug approval, for example, HER2, 5q-, CD20 ${ }^{+}$, estrogen receptor $(\mathrm{ER})^{+}$, EGFR, bcr-abl, KRAS and KIT. By understanding the drug, targets and pathways, we can predict toxicology, early proof of mechanism and determine the overall biologically effective dose. Predictive, prognostic and surrogate markers permit focused clinical studies with a higher probability of demonstrating clinical benefit through adaptive trial design and enabling cost-effective healthcare to personalize medicine. Biomarker development is hindered by the fact that additional biopsies or clinical trials may not reflect standard of medical

\section{Bibliography}

1 Miller VA, Riely GJ, Zakowski MF et al:: Molecular characteristics of bronchioalveolar carcinoma and adenocarcinoma, bronchioalveolar carcinoma subtype, predict response to erlotinib. J. Clin. Oncol. 26(9), 1472-1478 (2008).

2 Amado RG, Wolf M, Peeters $M$ et al:: Wild-type $K R A S$ is required for panitumumab efficacy in patients care and that economic incentives in industry for biomarker development are lacking. Evidence suggests, however, that the shortest development time of first-in-human to approval is with biomarker-associated targeted agents [4].

SNPs and copy-number variations may explain heterogeneity in cancer outcomes both as prognostic and predictive factors. Michael Gottesman reviewed drug resistance involving P-glycoprotein and the mechanism by which a SNP haplotype affects substrate specificity and inhibitor function, but not level of p-glycoprotein expression owing to delayed kinetics of translation. James Ford discussed pharmacogenetics/ genomics studies using candidate genes, candidate pathways, genome-wide association studies or large scale resequencing [5,101]. Examples of the predictive value of SNPs included thiopurine analogs in pediatric leukemia (thiopurine- $S$ methyltransferase [TPMT]), irinotecan in colon cancer (UDP-glyosyl transferase 1 [UGT1A1]), cisplatin (glutathion-S-transferase 4 [GSTM4]) and oxaliplatin (excision repair cross-complementation group 1 [ERCC1]) in lung cancer. Cost, practicality and ethical issues are not insignificant. However, the clinical promise is to preidentify genetic backgrounds likely to respond with decreased adverse effects.

\section{Financial \& competing interests disclosure}

The author has no relevant affiliations or financial involvement with any organization or entity with a financial interest in or financial conflict with the subject matter or materials discussed in the manuscript. This includes employment, consultancies, honoraria, stock ownership or options, expert testimony, grants or patents received or pending, or royalties.

No writing assistance was utilized in the production of this manuscript. with metastatic colorectal cancer. J. Clin. Oncol. 26(10), 1626-1634 (2008).

3 Sawyers CL: The cancer biomarker problem. Nature 452(7187), 548-552 (2008).

4 Goulart BH, Clark JW, Pien HH et al.: Trends in the use and role of biomarkers in Phase I oncology trials. Clin. Cancer Res. 13(22 Pt 1), 6719-6726 (2007).
5 Bodmer W, Bonilla C: Common and rare variants in multifactorial susceptibility to common diseases. Nat. Genet. $40(6), 695-701$ (2008).

\section{Website}

101 PharmaGKB: The Pharmacogenetics and Pharmacogenomics Knowledge Base http://pharmgkb.org 